\title{
Óleos essenciais no controle de Botrytis cinerea: influência na qualidade pós-colheita de uvas 'Rubi'
}

\author{
Essential oils in the control of Botrytis cinera: influence on \\ post harvest quality of Rubi grapes
}

\author{
Carla Garcia $^{1 *}$ (1), João Domingos Rodrigues², Sergio Miguel Mazaro³, \\ Renato Vasconcelos Botelho ${ }^{1}$, Cacilda Márcia Duarte Rios Faria ${ }^{1}$
}

\author{
${ }^{1}$ Universidade Estadual do Centro-Oeste (UNICENTRO), Departamento de Agronomia, Guarapuava/PR - Brasil \\ ${ }^{2}$ Universidade Estadual Paulista (UNESP), Departamento de Botânica, Botucatu/SP - Brasil \\ 3Universidade Tecnológica Federal do Paraná (UTFPR), Dois Vizinhos/PR - Brasil
}

*Corresponding Author: Carla Garcia, Universidade Estadual do Centro-Oeste (UNICENTRO), Departamento de Agronomia, Rua Simeão Varela de Sá, 03, CEP: 85040-080, Guarapuava/PR - Brasil, e-mail:

carlagarciaagro@gmail.com

Cite as: Garcia, C., Rodrigues, J. D., Mazaro, S. M., Botelho, R. V., \& Faria, C. M. D. R. (2019). Essential oils in the control of Botrytis cinera: influence on post harvest quality of Rubi grapes. Brazilian Journal of Food Technology, 22 e2018177. https://doi.org/10.1590/1981-6723.17718

\begin{abstract}
Resumo
Para que ocorra o controle de doenças na pós-colheita de uvas, são utilizados agroquímicos que, apesar de eficientes, podem causar problemas à saúde humana. Neste sentido, o presente trabalho tem o objetivo de verificar o potencial dos compostos voláteis presentes no óleo essencial de pitanga (OEP), guaçatonga (OEG) e melaleuca (OEM) no controle de Botrytis cinerea tanto in vitro quanto inoculados nas bagas de uvas 'Rubi', bem como verificar a indução de resistência nesses frutos. Dessa forma, avaliou-se o crescimento micelial do $B$. cinerea, o controle do mofo cinzento nas uvas 'Rubi', a qualidade pós-colheita dessas bagas e a atividade das enzimas polifenoloxidase (PPO), fenilalanina amônio liase (PAL), glucanase (GLU) e quitinase. De acordo com os resultados, observou-se que os compostos voláteis liberados pelos óleos essenciais reduziram os índices de velocidade de crescimento micelial de $B$. cinerea e da doença do mofo cinzento. Os tratamentos com voláteis de OEP e OEG ativaram mecanismos de indução de resistência desses frutos. Dessa forma, ressalta-se que OEP e OEG liberam compostos que apresentam a capacidade de agir diretamente sobre $B$. cinerea e também induzem a resistência de uvas 'Rubi', reduzindo o mofo cinzento nessas uvas.
\end{abstract}

Palavras-chave: Polifenoloxidase; Fenilalanina amônio liase; Glucanase; Quitinase.

\begin{abstract}
Agrochemicals are used to control diseases in post-harvest grapes, but although efficient, they may cause human health problems. Thus, the objective of the present work was to verify the potential of the volatile compounds present in the essential oils of the fruits pitanga (EOP), guaçatonga (EOG) and melaleuca (EOM) in the control of Botrytis cinerea both in vitro and inoculated in ' Rubi ' grapes, as well as to verify the induction of resistance in these fruits. Thus, $B$. cinerea mycelial growth, grey mould control in 'Rubi' grapes, post-harvest quality of the grapes and
\end{abstract}


the activities of the enzymes polyphenoloxidase (PPO), phenylalanine ammonium lyase (PAL), glucanase (GLU) and chitinase, were evaluated. According to the results it was observed that the volatile compounds released by the essential oils reduced the rates of mycelial growth of $B$. cinerea and of the grey mould disease, and the treatments with the volatiles from EOP and EOG activated the resistance induction mechanisms of these fruits. Thus, EOP and OEG release compounds that can act directly on $B$. cinerea and also induce resistance in the 'Rubi' grapes, reducing the grey mould in these grapes.

Keywords: Polyphenoloxidase; Phenylalanine ammonia lyase; Glucanase; Chitinase.

\section{Introdução}

As videiras 'Rubi' apresentam alta qualidade de seus frutos devido à capacidade de acúmulo de ácidos orgânicos, que interferem nas propriedades organolépticas de suas uvas, e de compostos fenólicos, como fenóis e antocianinas, que favorecem na qualidade de seus frutos. Porém, apresentam como entrave para o cultivo e a comercialização de seus cachos a baixa resistência a doenças (Pommer et al., 1997; Ferrandino et al., 2017).

Essa susceptibilidade às doenças, principalmente ao mofo cinzento (Botrytis cinerea), faz com que ocorra redução em sua produtividade e na qualidade pós-colheita desses frutos, devido aos danos desse patógeno na pré-colheita, durante o transporte ou no armazenamento das uvas (Camili et al., 2007). Como sintoma comum nas bagas, observa-se rachadura lateral, de coloração avermelhada, que evolui para mancha necrótica com esporulação do patógeno (Zoffoli et al., 2009).

São utilizados produtos químicos para o controle dessa doença, que acarretam problemas ambientais e à saúde humana. Como forma de eliminar ou ao menos reduzir esses impactos, tem-se buscado produtos alternativos que visam ao controle de doenças e limitem os riscos de ingestão e contaminação desses compostos químicos (Arruda et al., 2011).

Dentre esses produtos alternativos, estão os óleos essenciais, como os de pitanga (Eugenia uniflora), guaçatonga (Cacearia sylvestris) e melaleuca (Melaleuca alternifolia), por apresentarem compostos antimicrobianos, como o furanodieno (17,9\%) e o linalol (5,76\%), presentes no óleo essencial de pitanga; o cariofileno (86,6\%) e o pineno (2\%), no de guaçatonga, e o cineol (36\%), o limoneno (4\%) e o pineno (6\%), no de melaleuca (Jesus et al., 2015; Ferreira et al., 2011; Thambi et al., 2013).

Esses compostos apresentam potencial de atuar diretamente sobre o patógeno alterando o potencial transmembrana de suas células, reduzindo a síntese de ATP e, principalmente, por ocasionar danos no DNA e nas membranas das mitocôndrias (Bakkali et al., 2008). Esses fatos acarretam o controle do desenvolvimento de fungos fitopatogênicos devido a redução do crescimento micelial e redução da esporulação e da germinação dos esporos (Piati et al., 2017). Também se ressalta que componentes presentes nos óleos essenciais possuem a capacidade de ativar mecanismos de defesa das plantas e, assim, pode-se obter a resistência contra patógenos (Mazaro et al., 2008).

Dessa forma, o trabalho teve como objetivo analisar se os compostos voláteis exalados por esses óleos apresentam a capacidade de controlar o desenvolvimento de Botrytis cinerea in vitro e do mofo cinzento na pós-colheita de bagas de videira 'Rubi', verificando se o procedimento interfere na qualidade pós-colheita dessas uvas e se há ativação de enzimas de defesa no fruto.

\section{Material e métodos}

Os óleos utilizados nos experimentos foram cedidos pela Universidade Tecnológica Federal do Paraná (UTFPR) - Campus Dois Vizinhos, oriundos da Empresa Garden City. 
Os experimentos foram realizados no Laboratório de Fitopatologia e de Análises de Pós-Colheita de Frutas e Hortaliças da Universidade Estadual do Centro-Oeste.

Para verificar o efeito dos voláteis liberados pelos óleos essenciais no crescimento micelial de $B$. cinerea, utilizou-se meio de cultura BDA (Batata-Ágar-Dextrose) autoclavado por 20 minutos a $120{ }^{\circ} \mathrm{C}$ sob pressão de $1 \mathrm{~atm}$ vertido em placas de Petri, adicionando-se, em seguida, um disco de 1,5 cm de diâmetro de micélio do patógeno no centro de cada placa. Do lado oposto ao meio de cultura, ou seja, na tampa, foi colocada fita dupla face para fixar $1 \mathrm{~cm}^{2}$ de papel germitest, no qual foram pipetados $20 \mu \mathrm{L}$ dos tratamentos com água (tratamento testemunha) e os óleos essenciais de pitanga, guaçatonga e melaleuca a $100 \%$. Em seguida, as placas foram acondicionadas em sala climatizada com temperatura de $25^{\circ} \mathrm{C}$ e fotoperíodo de 12 horas. Com o intuito de avaliar o tempo de contato dos compostos voláteis presentes nos tratamentos com o patógeno, parte do experimento ficou em contato por uma hora e outra por 96 horas.

Após 24 horas, avaliou-se diariamente o crescimento micelial pelo diâmetro da colônia, até que o tratamento testemunha crescesse em toda a placa, totalizando quatro avaliações. Esses dados foram utilizados no cálculo do índice de velocidade de crescimento micelial (IVCM) (Araújo et al., 2008).

Para verificar o controle do mofo cinzento, utilizaram-se uvas 'Rubi', enxertadas sobre IAC-766 no ciclo do ano de 2015 (safra julho/agosto) e colhidas dia 07/julho em vinhedo comercial localizado no município de Marialva-PR. Em seguida, os frutos foram armazenados em caixas de papelão ondulado, transportados de forma refrigerada para a cidade de Guarapuava-PR (284 km distante) e armazenados em câmara fria $\left(1 \pm 2{ }^{\circ} \mathrm{C}\right.$ e 95\%UR) por 19 dias, quando estas uvas foram selecionadas para a condução dos experimentos.

Posteriormente, separaram-se quatro bagas por cacho e foram colocadas cinco dessas estruturas (parcela experimental) em bandejas de poliestireno (polímero de estireno) com espessura de $30 \mathrm{~mm}$ e tamanho de $35 \times 23 \times 33 \mathrm{~cm}$, contendo no centro $2 \mathrm{~cm}^{2}$ de papel germitest com uma alíquota de $20 \mu \mathrm{L}$ dos tratamentos com água (testemunha) e os óleos essenciais de pitanga (OEP), guaçatonga (OEG) e melaleuca (OEM), todos a $100 \%$. Posteriormente, essas bandejas foram fechadas com saco plástico transparente de polietileno $(30 \times 40 \mathrm{~cm})$ e acondicionadas em sala climatizada a $25^{\circ} \mathrm{C}$. Após uma hora de contato dos tratamentos com as bagas, retirou-se o papel germinest do interior das embalagens. Também se adotou a mesma metodologia para verificar o efeito dos tratamentos por 13 dias, período necessário para o desenvolvimento e a avaliação do mofo cinzento nas uvas.

Após 24 horas, foi realizada a coleta de duas bagas para a análise de enzimas, as quais, em seguida, foram acondicionadas em freezer a $-20{ }^{\circ} \mathrm{C}$. Posteriormente, foi inoculada suspensão de $B$. cinerea a $2,2 \times 10^{5}$ conídios $\mathrm{mL}^{-1}$ nas demais bagas. Após seis dias, iniciaram-se as avaliações diárias do índice de doença (\%) do mofo cinzento, de acordo com a escala de nota de Camili et al. (2007), por cinco dias. No último dia de avaliação, duas bagas de cada parcela experimental foram transferidas para freezer a $-20{ }^{\circ} \mathrm{C}$ para determinação de compostos fenólicos e análise da atividade enzimática, e as demais foram utilizadas nas análises pós-colheita.

As bagas destinadas às análises pós-colheita foram trituradas em liquidificador e, a partir desse mosto, determinou-se o teor de sólidos solúveis (SS), a acidez titulável (AT) (titulação com solução padronizada de $\mathrm{NaOH}$ com ponto final de pH 8,2) e "ratio", relação SS/AT (Instituto Adolfo Lutz, 1985).

Para determinação do teor de compostos fenólicos, seguiu-se a metodologia de extração de Rossi Junior \& Singleton (1965), conforme Association of Official Analytical Chemists (1992).

Para verificar a atividade das enzimas de indução de resistência das uvas 'Rubi', utilizou-se a forma de extração descrita por Guzzo \& Martins (1996). O extrato enzimático foi utilizado para a determinação do teor de proteínas totais (Bradford, 1976), atividade específica de polifenoloxidase (PPO) (Gauillard et al., 1993), fenilalanina amônio liase (PAL) (Rodrigues et al., 2006), glucanase (GLU) e quitinase descrita por Guzzo \& Martins (1996). 
O delineamento experimental de todos os experimentos descritos neste trabalho, foram inteiramente casualizado em esquema fatorial $3 \times 2$ (três tipos de óleos essenciais em dois tempos de contato) com quatro repetições, todos instalados em duplicatas. Dessa forma, os resultados apresentados são referentes à média dos dois experimentos de cada análise.

Os dados foram submetidos à análise de variância (teste F), sendo as médias comparadas pelo teste Tukey no nível de 5\% de probabilidade, utilizando o programa estatístico Sisvar (Ferreira, 2011).

\section{Resultados e discussão}

Com relação ao índice de velocidade de crescimento micelial (IVCM) de B. cinerea, verificou-se interação entre os óleos essenciais e o tempo de contato com os tratamentos. Ressalta-se que o OEM reduziu em 43,1 e 68,8\%, respectivamente, em relação à testemunha para os tempos de exposição de 1 h e 96 h (Figura 1). Destaca-se também que, na comparação entre esses tempos, o OEM diminuiu em 48,3\% o IVCM (Figura 1A).

Com relação ao índice de doença (ID) do mofo cinzento, observou-se que o OEM induziu em 21,2\% e o OEP e OEG reduziram em 45,7 e 43,1\%, respectivamente, em relação ao tratamento testemunha (Figura 1B).
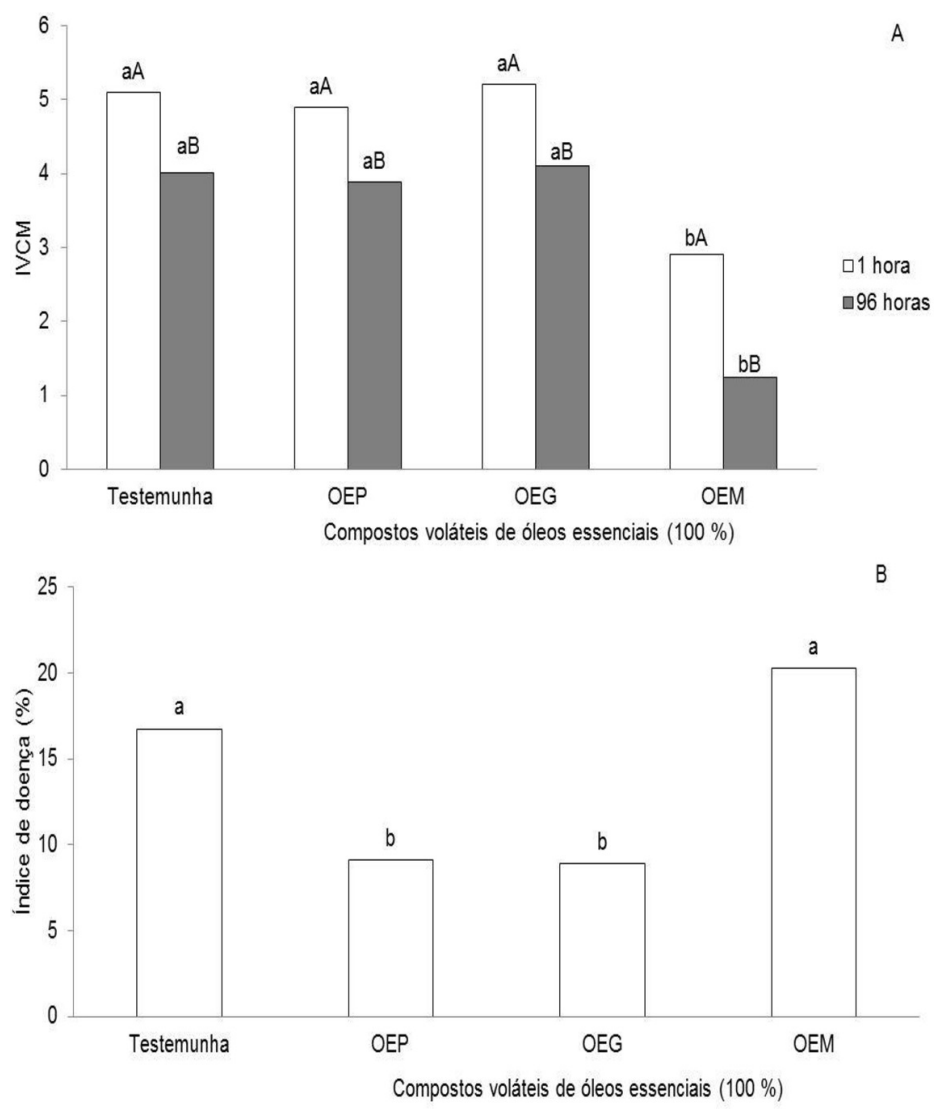

Figura 1. Índices de velocidade de crescimento micelial (IVCM) de Botrytis cinerea (A) e de doença de mofo cinzento (B), sob efeito dos compostos voláteis dos óleos essenciais de pitanga (OEP) (Eugenia uniflora), guaçatonga

(Casearia sylvestris) (OEG) e melaleuca (OEM) (Melaleuca alternifolia) a 100\%. Comparação entre todos os tratamentos. Letras minúsculas estão relacionadas com os tratamentos e as maiúsculas entre os tempos.

Resultados referentes aos voláteis eliminados pelo OEP podem-se relacionar com a capacidade de seus compostos em causar alterações degenerativas nas hifas, acarretando aumento nos vacúolos, elevado número de peroxissomos e de mitocôndrias nas células fúngicas, e perda da integridade da membrana plasmática (Pârvu et al., 2013; Shao et al., 2013). Quando as plantas entram em contato com algum patógeno, 
naturalmente emitem compostos voláteis ricos em monoterpenos e sesquiterpenos. Videiras resistentes ao Plasmopara viticola liberam, principalmente muroleno, cariofileno e germacreno B, como forma de proteção (Lazazzara et al., 2018). Esses compostos são encontrados no OEP e em OEG, os quais, possivelmente, foram os responsáveis pela redução do mofo cinzento nas bagas de uva 'Rubi' .

Além desses efeitos proporcionados pelo OEP, ressalta-se que esse óleo essencial apresenta volatilização lenta, o que permite a formação de envelopes de proteção sobre o tecido vegetal (Lazazzara et al., 2018), fato que, possivelmente, protegeu os frutos de uvas 'Rubi'.

A retenção dos voláteis desses agentes antimicrobianos aumenta no interior de embalagens de polietileno (Lou et al., 2017). Porém, para o OEM, esse fato não foi observado, possivelmente devido à sua alta volatilização, que reduziu sua capacidade de controle do mofo cinzento nas uvas 'Rubi' (Souza et al., 2017).

De acordo com os resultados relacionados com a pós-colheita das uvas 'Rubi', os dados não apresentaram interação entre tratamento e o tempo de contato. Observou-se que, para o teor de sólidos solúveis (SS), o OEP e o OEM aumentaram essa variável em $20 \%$ e $27,1 \%$, respectivamente, em relação ao tratamento testemunha (Figura 2A). Para acidez titulável (AT), OEP, OEG e OEM aumentaram em 5\%, 5,5\% e 6\% e, para o ratio (relação SS/AT), o aumento foi de $59 \%, 52 \%$ e $37 \%$, respectivamente, todos em relação ao tratamento testemunha (Figuras 2B e 2C).

A concentração de compostos fenólicos foi reduzida em 19,1\% e 16,9\%, respectivamente, pelos tratamentos com OEG e OEM, porém verificou-se que OEM não apresentou diferença estatística com a testemunha e também se mostrou similar ao OEP. Verificou-se, ainda, que OEM induziu em 3,4\%, quando os tratamentos foram comparados entre si (Figura 2D).
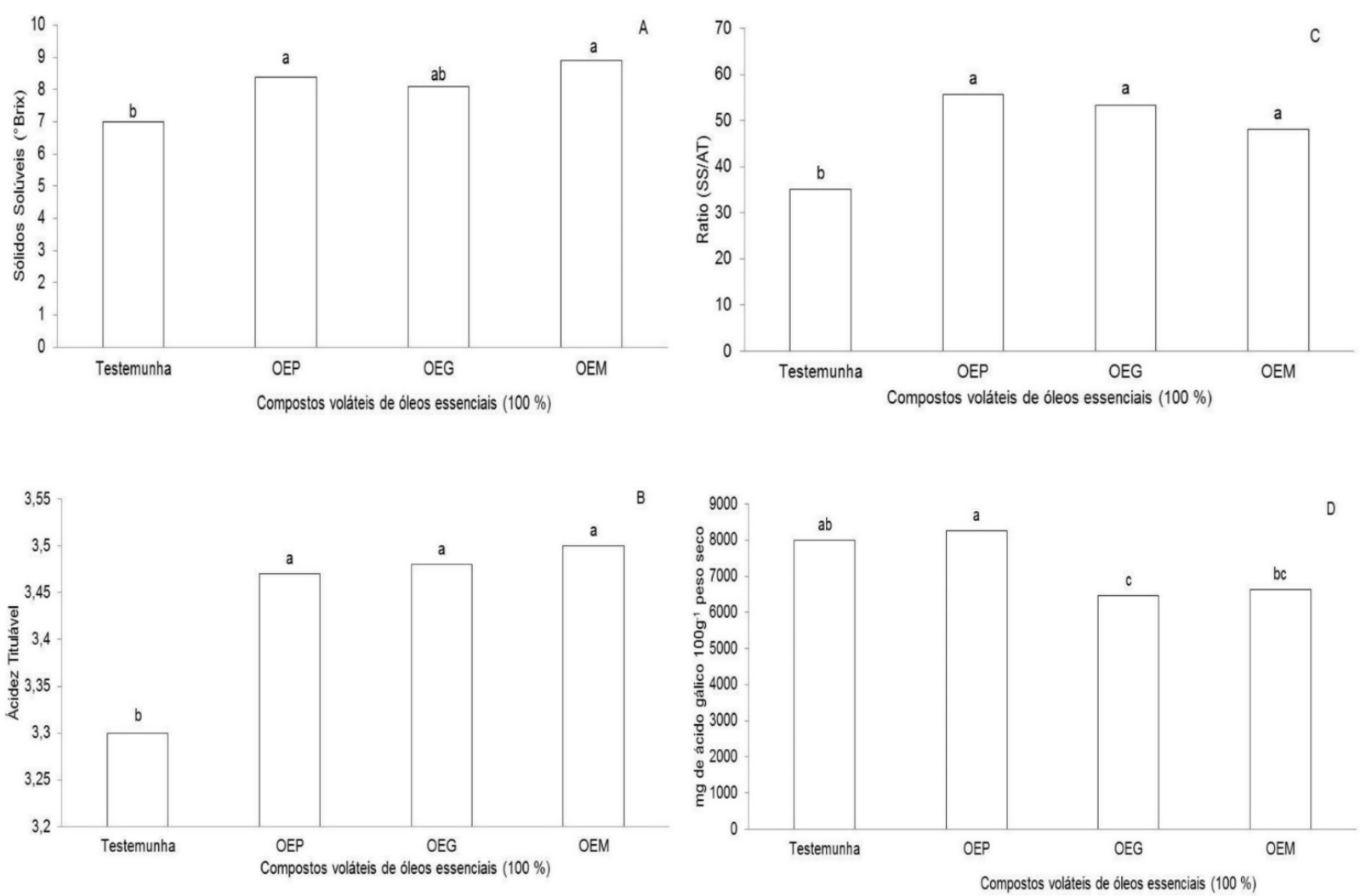

Figura 2. Análises de pós-colheita do teor de sólidos solúveis ( $\left.{ }^{\circ} \mathrm{Brix}, \mathrm{SS}\right)(\mathrm{A})$, acidez titulável (\%, AT) (B), Ratio (relação SS/AT) (C) e teor de compostos fenólicos (mg de ácido gálico $100 \mathrm{~g}^{-1}$ de massa seca) (D) em mostro de bagas de uvas 'Rubi' tratadas com compostos voláteis dos óleos essenciais de pitanga (OEP) (Eugenia uniflora), guaçatonga (OEG) (Casearia sylvestris) e melaleuca (OEM) (Melaleuca alternifolia) a 100\%. 
O contato dos compostos voláteis presentes em OEP, OEG e OEM melhorou a qualidade pós-colheita das uvas 'Rubi', proporcionando sabor adocicado aos frutos, com redução na acidez e elevada qualidade, o que irá propiciar maior aceitabilidade no mercado (Moraes et al., 2008; Domingues Neto et al., 2016). Dessa forma, pode-se dizer que esses óleos essenciais apresentam alto potencial para serem adicionados nas embalagens para comercialização desses frutos. Deve-se levar em consideração que a forma de armazenamento é que irá determinar a qualidade com que essas uvas de mesa irão chegar até o consumidor (Rasulov, 2017).

OEP induziu a produção de elevado teor de compostos fenólicos, porém não apresentou diferença estatística com a testemunha. As plantas apresentam normalmente altas concentrações desses compostos, explicando os resultados obtidos no mosto das bagas do tratamento testemunha (Shalaby \& Horwitz, 2015). Essa indução desencadeada pelo OEP está relacionada com a capacidade de os compostos fenólicos apresentarem efeito tóxico sobre micro-organismos ou atuarem na deposição de lignina para o fortalecimento da parede celular desses frutos (Andreasson et al., 2017). Esses fatos colaboraram para a redução do índice do mofo cinzento nas bagas de uvas 'Rubi' tratadas com OEP, porém, na testemunha, foram insuficientes para desencadear esse efeito.

Além dessas funções, os compostos fenólicos são importantes para a saúde humana. Previnem arteriosclerose, diabetes e doenças neurodegenerativas, inibem o crescimento de células cancerígenas e são anti-inflamatórios, analgésicos, gastroprotetores e antimicrobianos (Cetin-Karaca \& Newman, 2015; Lajili et al., 2016).

Com relação à atividade da enzima polifenoloxidase (PPO), não houve interação entre os fatores e observou-se que sua atividade foi inibida em $51,6 \%$ e $47,7 \%$ pelos tratamentos com os compostos voláteis de OEG e OEM, respectivamente, antes da inoculação do patógeno, B. cinerea (Figura 3A). A atividade depois dessa inoculação também foi reduzida por todos os óleos essenciais, mas, significativamente, pelo OEM, em 78,9\% em relação ao tratamento testemunha (Figura 3B).
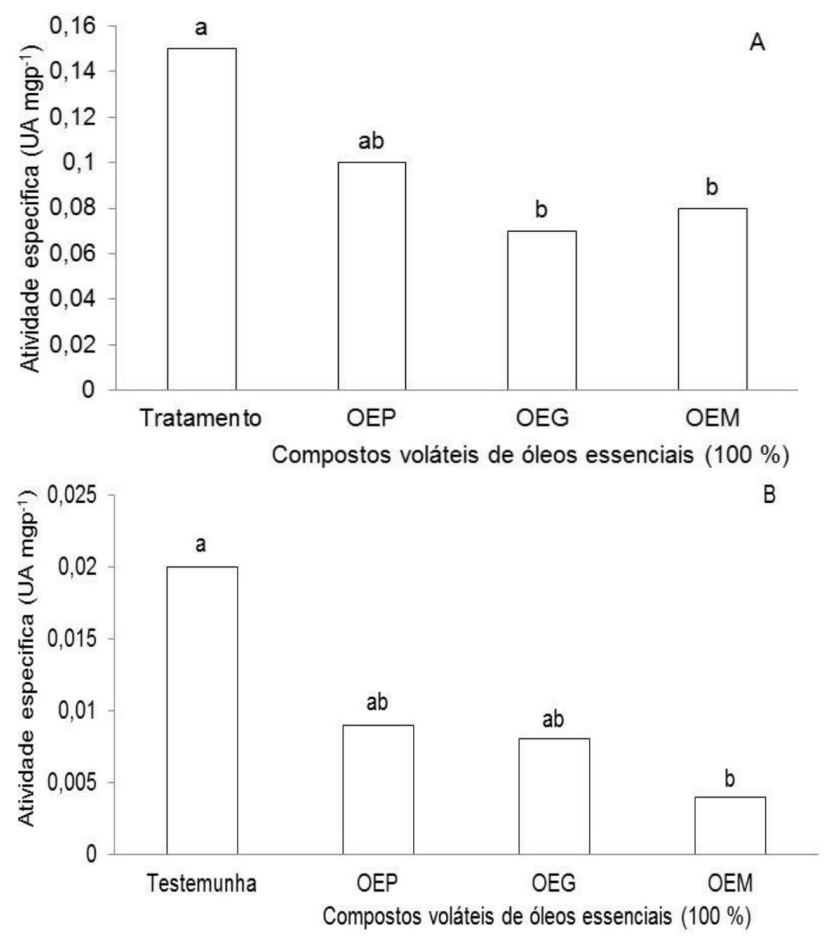

Figura 3. Atividade específica da polifenoloxidase (PPO) antes (A) e após (B) a inoculação de Botrytis cinerea em bagas de uvas 'Rubi' tratadas com compostos voláteis dos óleos essenciais de pitanga (OEP) (Eugenia uniflora), guaçatonga (OEG) (Casearia sylvestris) e melaleuca (OEM) (Melaleuca alternifolia) a 100\%. 
A elevada atividade de PPO indica a rápida deterioração de uvas 'Rubi' (Freitas et al., 2008), fato que foi observado somente no tratamento testemunha, enquanto nos demais ocorreu o inverso (Figura 3).

Os tratamentos com voláteis de OEG e OEM reduziram o teor de compostos fenólicos (Figura 2D) nas células dessas uvas. Esse fato dificulta a atividade da PPO, pois essa enzima promove a oxidação enzimática desses compostos, formando quinonas. Dessa forma, a presença desses substratos enzimáticos torna os frutos susceptíveis à reação desencadeada pela PPO, resultando no escurecimento do tecido da baga. Inicialmente, as quinonas podem rapidamente se condensar, formando pigmentos insolúveis e escuros denominados melaninas, ou reagirem, de forma não enzimática, com aminoácidos, proteínas ou outros compostos (Menolli et al., 2008; Zhang et al., 2016).

O escurecimento dos frutos acarreta efeitos negativos na coloração e no sabor das bagas de uva (Ghasemzadeh et al., 2016). Para Zimdars et al. (2017), a intensidade dos impactos na extensão da deterioração da cor depende da atividade das enzimas também secretadas por $B$. cinerea. Nesse caso, os compostos voláteis dos óleos essenciais reduziram a atividade dessa enzima, indicando que os frutos apresentam maior qualidade em relação à testemunha.

Para os resultados da atividade da enzima PAL, verificou-se que as coletas, antes e após a inoculação de B. cinerea nas uvas 'Rubi', apresentaram interação entre os fatores. É interessante ressaltar que, na coleta antes da inoculação do patógeno, os tratamentos com os óleos essenciais induziram a atividade de PAL, observando-se aumentos de 99,7\%, 298,7\% e 115,6\% para OEP, OEG e OEM, respectivamente, no período de uma hora das uvas em contato com os compostos voláteis desses óleos. Com relação aos 13 dias, OEP e o OEG aumentaram a atividade da PAL em 916,1\% e 109,1\%, respectivamente, porém OEM inibiu em 100\% a sua atividade, em relação ao tratamento testemunha. Quando se comparou o tempo de contato de uma hora e 13 dias para as uvas tratadas com OEP, observou-se aumento de 384,5\% na atividade dessa enzima nas uvas que ficaram por tempo maior de exposição aos compostos voláteis desse óleo essencial. Já para OEG, nessa mesma comparação, a atividade da PAL dobrou em uma hora, enquanto para OEM esse incremento foi de $100 \%$ (Figura $4 \mathrm{~A}$ ).

Com relação à coleta após a inoculação do patógeno, verificou-se que OEP e OEG reduziram a atividade da PAL, sendo que OEM não apresentou diferença estatística com a testemunha. Porém, observou-se que, para 13 dias, OEM induziu a atividade dessa enzima em 6.000 vezes em relação ao tempo de exposição de uma hora com os tratamentos (Figura 4B). 

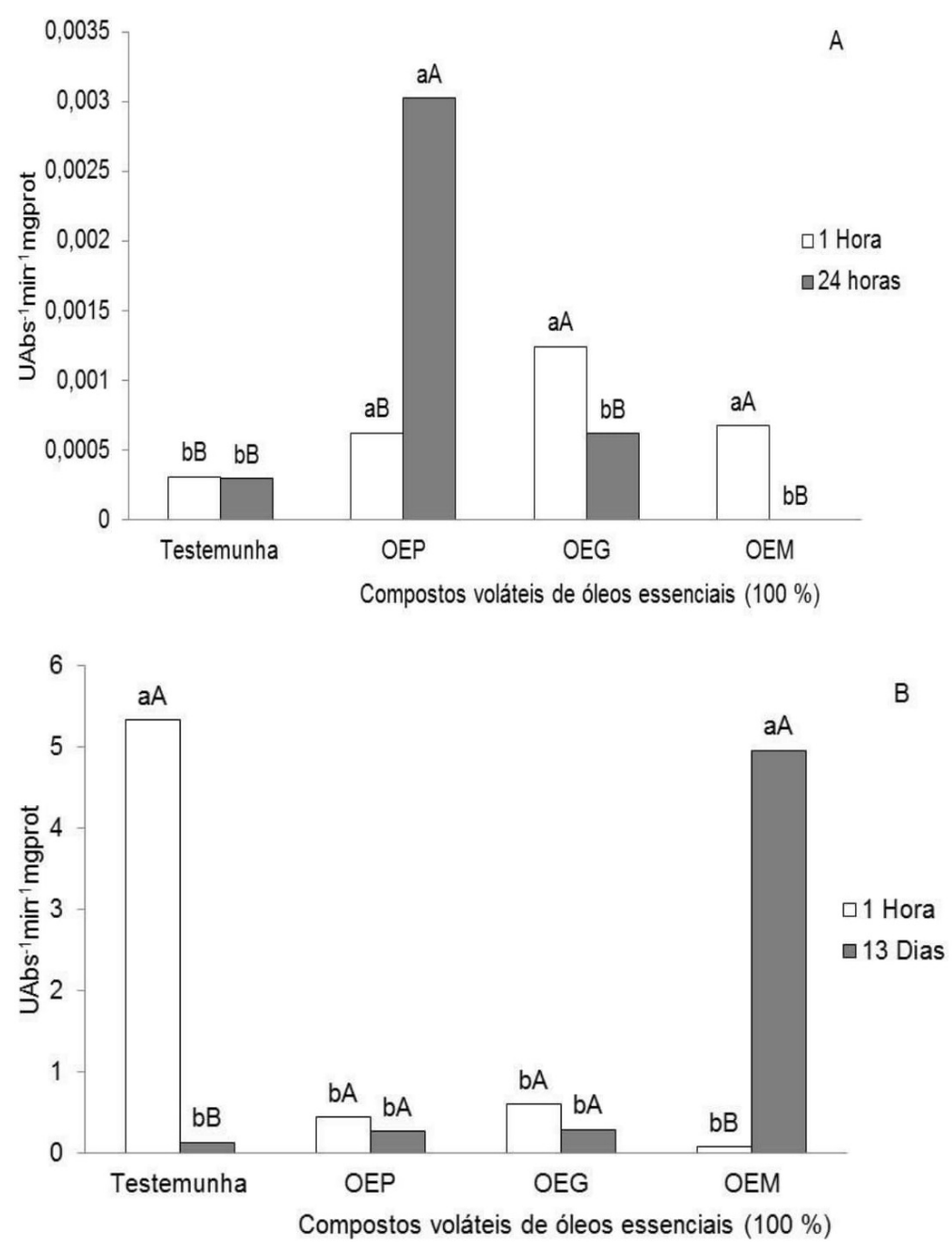

Figura 4. Atividade específica da fenilalanina amônio liase (PAL) antes (A) e após (B) a inoculação de Botrytis cenerea, em bagas de uvas 'Rubi' tratadas com compostos voláteis dos óleos essenciais de pitanga (OEP) (Eugenia uniflora), guaçatonga (OEG) (Casearia sylvestris) e melaleuca (OEM) (Melaleuca alternifolia) a 100\%, após uma hora e 13 dias de contato. Comparação entre todos os tratamentos. Letras minúsculas estão relacionadas com os tratamentos e as maiúsculas entre os tempos.

A enzima PAL faz parte da via dos fenilpropanoides, que utilizam cerca de $40 \%$ do carbono orgânico biosférico na síntese de lignina depositada na parede celular (Zhang \& Liu, 2015). De acordo com essa observação, pode-se dizer que a indução dessa enzima, estimulada por OEP antes da inoculação de $B$. cinerea, favoreceu a redução do mofo cinzento nas bagas, devido à deposição de lignina na parede celular e, consequentemente, aumentando a resistência das células dos frutos à entrada do patógeno.

De acordo com os resultados verificados pelos voláteis do OEM, verificou-se comportamento semelhante ao da testemunha, ou seja, indução da atividade da PAL apenas após o contato com o patógeno. Assim, verifica-se que os estímulos para síntese de lignina foram tardios para dificultar a infecção de $B$. cinerea nesses frutos.

A rota de ativação de síntese da lignina via PAL resulta na formação de monolignóis que serão utilizados na deposição de lignina na parede celular. A restrição da atividade dessa enzima diminui a concentração de lignina nas células; como consequência, o fruto mostra-se suscetível ao patógeno fúngico (Cass et al., 2015).

Para Danner et al. (2008), a redução da área lesionada em pêssegos infectados por Monilinia fruticola está relacionada à atividade da PAL. Além de proporcionar a resistência da parede celular através da desaminação de L-fenilalanina em ácido transcinâmico, essa enzima proporciona, como consequência desse processo, a 
síntese de fitoalexinas e fenóis que também dificultam a entrada de fungos nos tecidos (Gholizadeh \& Kohnehrouz, 2010).

Os resultados da atividade da glucanase antes da inoculação de $B$. cinerea não apresentaram interação entre os fatores. Destaca-se que OEM induziu a atividade em $27,7 \%$ em relação ao tratamento testemunha (Figura 5A).

A coleta realizada após a inoculação do patógeno apresentou interação entre os fatores, destacando-se que não foram observadas diferenças estatísticas entre os tratamentos quando as uvas ficaram por uma hora em contato com seus compostos voláteis. Porém, com 13 dias, OEM induziu a atividade da GLU em aproximadamente 20 mil vezes quando comparada sua atividade nas bagas com uma hora em contato com esse óleo essencial (Figura 5B).
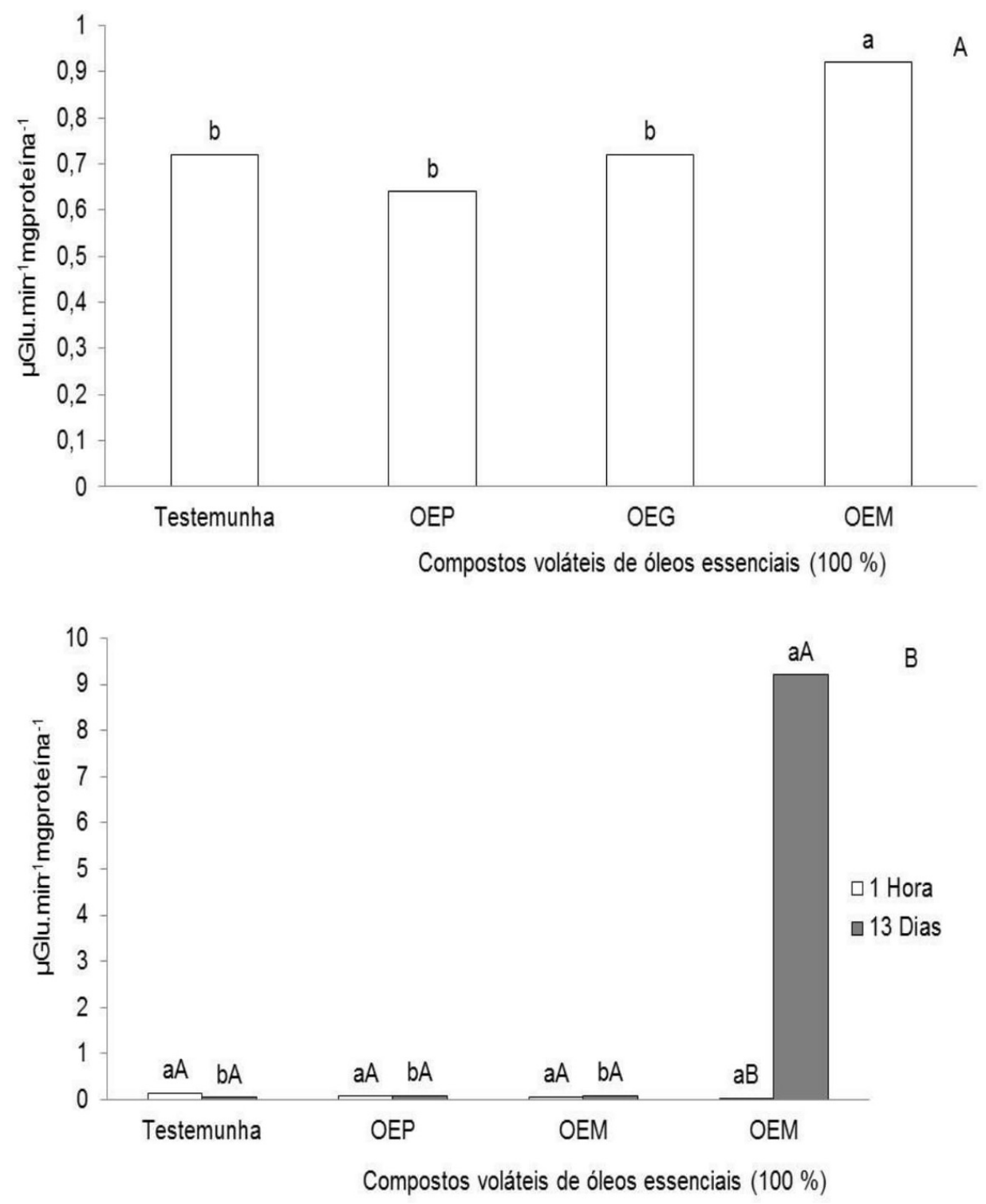

Figura 5. Atividade específica de glucanase (GLU), antes (A) e após (B) a inoculação de Botrytis cenerea, em bagas de uvas 'Rubi' tratadas com compostos voláteis dos óleos essenciais de pitanga (OEP) (Eugenia uniflora), guaçatonga (OEG) (Casearia sylvestris) e melaleuca (OEM) (Melaleuca alternifolia) a 100\%, após uma hora e 13 dias de contato.

Comparação entre todos os tratamentos. Letras minúsculas estão relacionadas com os tratamentos e as maiúsculas entre os tempos. 
As cascas de uvas 'Rubi' não apresentaram atividade da enzima quitinase antes da inoculação de B. cinerea. Após a inoculação do patógeno, verificou-se interação entre os fatores tratamentos e tempos de exposição. Ressalta-se que todos os tratamentos reduziram acima de, aproximadamente, $76 \%$ a atividade dessa enzima em relação ao tratamento testemunha (Figura 6).

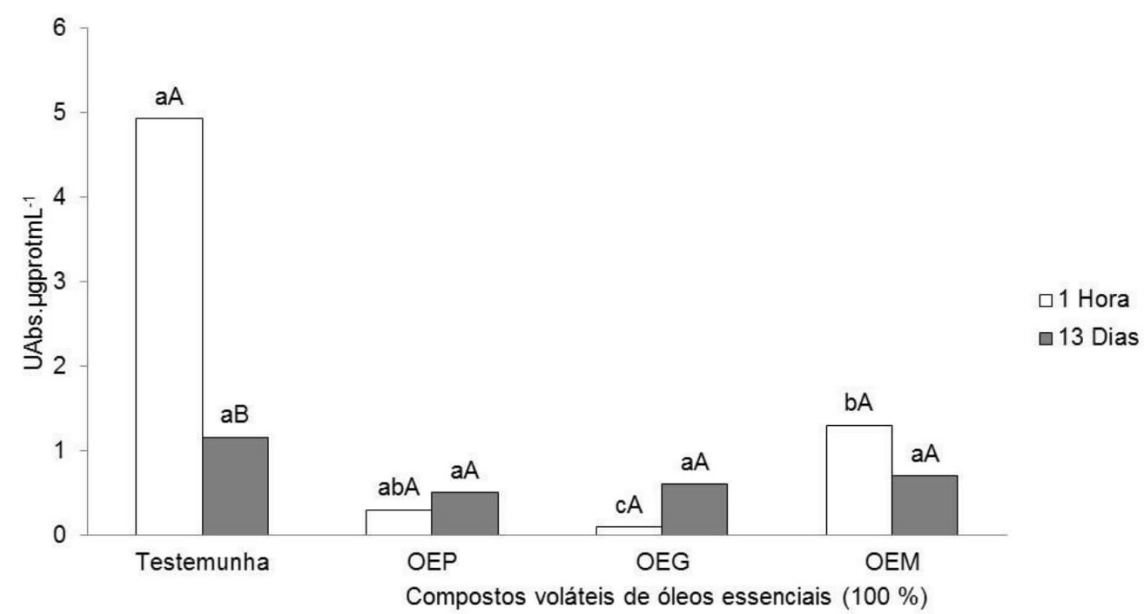

Figura 6. Atividade específica de quitinase após a inoculação de Botrytis cenerea em bagas de uvas 'Rubi' tratadas com compostos voláteis dos óleos essenciais de pitanga (OEP) (Eugenia uniflora), guaçatonga (OEG) (Casearia sylvestris) e melaleuca (OEM) (Melaleuca alternifolia) a 100\%, após uma hora e 13 dias de contato. Comparação entre todos os tratamentos. Letras minúsculas estão relacionadas com os tratamentos e as maiúsculas entre os tempos.

Os compostos voláteis do OEM elevaram a atividade de glucanase nas bagas de uvas 'Rubi', porém essa indução não foi suficiente para reduzir o índice do mofo cinzento nas bagas. Essa enzima catalisa a clivagem das ligações $\beta$-1,3-glucosídicas em $\beta$-1,3-glucanos, que são considerados um dos principais componentes da parede celular de fungos fitopatogênicos. Com isso, a GLU hidrolisa as paredes desses micro-organismos, que atuam como elicitores de oligossacarídeos na ativação dos mecanismos de defesa do fruto (Keen \& Yoshikawa, 1983; Garfoot et al., 2017).

Todo esse mecanismo desencadeado pelo OEM, possivelmente, não foi eficaz, pois a parede celular de B. cinerea é composta por quitinas (Romani, 2011). Nesse caso, os tratamentos deveriam ter induzido a atividade da quitinase para que houvesse a degradação de quitinas e liberação de oligossacarídeos que atuariam como elicitores (Pascholati et al., 2008).

Esse fato é possível por causa da catalisação de clivagem que as quitinases realizam entre os carbonos 1 e 4 de dois monômeros de acetil-D-glucosamina de quitina. Também essas enzimas podem bloquear o crescimento das hifas, apresentando atividade antimicrobiana (Neuhaus et al., 1996; Van Loon, 2006).

Sellamuthu et al. (2013) ressaltam que vapores utilizados do óleo essencial de tomilho (Thymus vulgaris L.) reduzem a antracnose (Colletrotrichum gloeosporioides) em frutos de abacate. Segundo os autores, esse fato é possível devido à ação elicitora desses compostos voláteis em ativar respostas bioquímicas nesses frutos.

Dessa forma, ressalta-se que os compostos voláteis produzidos pelos óleos essenciais de pitanga, guaçatonga e melaleuca são eficazes para favorecer a qualidade pós-colheita de uvas 'Rubi'. Porém, o OEP se destaca por também reduzir o índice de mofo cinzento nas bagas, devido à ativação dos mecanismos de defesa da via dos fenilpropanoides com a atividade elevada da PAL. Com isso, pode-se sugerir o emprego desse tratamento no interior de embalagens de poliestireno para a comercialização dessas uvas. Porém, recomenda-se que testes de análises nutricionais sejam realizados nesses frutos. Todas essas características atendem às afirmações de Sivakumar \& Bautista-Baños (2014) de que as embalagens dos frutos devem conter fungicidas naturais com o intuito de reduzir o uso de moléculas sintéticas e tóxicas ao ser humano. Assim, pode-se enfatizar que os voláteis do OEP não apenas atuam como fungicidas, mas também como indutores de resistência. 


\section{Conclusões}

Os compostos voláteis do OEP melhoram a qualidade pós-colheita de uvas 'Rubi', reduzindo o mofo cinzento e induzindo mecanismos de defesa contra B. cinerea.

\section{Refêrencias}

Andreasson, E., Marit, L., \& Laith, M. (2017). Plant extracts comprising at least a phenolic compound, for inducing the natural defense of a plant against a pathogen, such as Phytophthora infestans. United States patent US9681669B2.

Araújo, L., Stadnik, M. J., Borsato, L. C., \& Valdebenito-Sanhueza, R. M. (2008). Fosfito de potássio e ulvana no controle da mancha foliar da gala em macieira. Tropical Plant Pathology, 33(2), 148-152. http://dx.doi.org/10.1590/S198256762008000200009

Arruda, M. C., Jacomino, A. P., Trevisan, M. J., Jeronimo, E. M., \& Moretti, C. L. (2011). Atmosfera modificada em laranja 'Pêra' minimamente processada. Bragantia, 70(3), 664-671. http://dx.doi.org/10.1590/S0006-87052011000300023

Association of Official Analytical Chemists - AOAC. (1992). Official Methods of Analysis of the Association of Official Analytical Chemists (12th ed.). Washington: AOAC.

Bakkali, F., Averbeck, S., Averbeck, D., \& Idaomar, M. (2008). Biological effects of essential oil: A review. Food and Chemical Toxicology, 46(2), 446-475. PMid:17996351. http://dx.doi.org/10.1016/j.fct.2007.09.106

Bradford, M. M. (1976). A rapid and sensitive method for the quantitation of microgram quantities of protein utilizing the principle of protein-dye binding. Analytical Biochemistry, 72(1-2), 248-254. PMid:942051. http://dx.doi.org/10.1016/0003-2697(76)90527-3

Camili, E. C., Benato, E. A., Pascholati, S. F., \& Cia, P. (2007). Avaliação de quitosana, aplicada em pós-colheita, na proteção de uva 'Itália' contra Botrytis cinerea. Summa Phytopathologica, 33(3), 215-221. http://dx.doi.org/10.1590/S010054052007000300001

Cass, C. L., Peraldi, A., Dowd, P. F., Mottiar, Y., Santoro, N., Karlen, S. D., Bukhman, Y. V., Foster, C. E., Thrower, N., Bruno, L. C., Moskvin, O. V., Johnson, E. T., Willhoit, M. E., Phutane, M., Ralph, J., Mansfield, S. D., Nicholson, P., \& Sedbrook, J. C. (2015). Effects of phenylalanine ammonia lyase (PAL) knockdown on cell wall composition, biomass digestibility, and biotic and abiotic stress responses in Brachypodium. Journal of Experimental Botany, 66(14), 4317-4335. PMid:26093023. http://dx.doi.org/10.1093/jxb/erv269

Cetin-Karaca, H., \& Newman, M. C. (2015). Antimicrobial efficacy of plant phenolic compounds against Salmonella and Escherichia Coli. Food Bioscience, 11, 8-16. http://dx.doi.org/10.1016/j.fbio.2015.03.002

Danner, M. A., Sasso, S. A. Z., Medeiros, J. G. S., Marchese, J. A., \& Mazaro, S. M. (2008). Indução de resistência à podridãoparda em pêssegos pelo uso de eliciadores em pós-colheita. Pesquisa Agropecuária Brasileira, 43(7), 793-799. http://dx.doi.org/10.1590/S0100-204X2008000700002

Domingues Neto, F. J., Pimentel Junior, A., Maia Paiva, A. P., Romano Modesto, L., \& Tecchio, M. A. (2016). Características físicas e físico-químicas da uva 'Rubi'(Vitis vinifera L.) cultivada em região subtropical. Revista Iberoamericana de Tecnología Postcosecha, 17(2), 262-266.

Ferrandino, A., Pagliarani, C., Carlomagno, A., Novello, V., Schubert, A., \& Agati, G. (2017). Improved fluorescence-based evaluation of flavonoid in red and white winegrape cultivars. Australian Journal of Grape and Wine Research, 23(2), $207-214$. http://dx.doi.org/10.1111/ajgw.12276

Ferreira, D. F. (2011). SISVAR: A computer statistical analysis system. Ciência e Agrotecnologia, 35(6), 1039-1042. http://dx.doi.org/10.1590/S1413-70542011000600001

Ferreira, P. M. P., Costa-Lotufo, L. V., Moraes, M. O., Barros, F. W. A., Martins, A. M. A., Cavalheiro, A. J., Bolzani, V. S. Santos, A. G., \& Pessoa, C. (2011). Folk uses and pharmacological properties of Casearia sylvestris: A medicinal review. Anais da Academia Brasileira de Ciências, 83(4), 1373-1384. PMid:22159347. http://dx.doi.org/10.1590/S0001-37652011005000040

Freitas, A. A. D., Francelin, M. F., Hirata, G. F., Clemente, E., \& Schmidt, F. L. (2008). Atividades das enzimas peroxidase (POD) e polifenoloxidase (PPO) nas uvas das cultivares benitaka e rubi e em seus sucos e geléias. Food Science and Technology, 28(1), 172-177. http://dx.doi.org/10.1590/S0101-20612008000100025

Garfoot, A. L., Dearing, K. L., VanSchoiack, A. D., Wysocki, V. H., \& Rappleye, C. A. (2017). Eng1 e Exg8 são as principais $\beta$ glucanases secretadas pelo patógeno fungo Histoplasma capsulatum. The Journal of Biological Chemistry, 292(12), 48014810. PMid:28154008. http://dx.doi.org/10.1074/jbc.M116.762104

Gauillard, F., Richard-Forget, F., \& Nicolas, J. (1993). New spectrophotometric assay for polyphenol oxidase activity. Activity Biochemistry, 215(1), 59-65. PMid:8297016.

Ghasemzadeh, A., Jaafar, H. Z., \& Rahmat, A. (2016). Variation of the phytochemical constituents and antioxidant activities of Zingiber officinale var. rubrum Theilade associated with different drying methods and polyphenol oxidase activity. Molecules, 21(6), 780. PMid:27322227. http://dx.doi.org/10.3390/molecules21060780

Gholizadeh, A., \& Kohnehrouz, B. B. (2010). Activation of phenylalanine ammonia lyase as a key component of the antioxidative system of salt-challenged maize leaves. Brazilian Journal of Plant Physiology, 22(4), 217-223. http://dx.doi.org/10.1590/S167704202010000400001

Guzzo, S. D., \& Martins, E. M. F. (1996). Local and systemic induction of $\beta-1,3-$ glucanase and chitinase in coffee leaves protected against Hemileia vastatrix by Bacillus thuringiensis. Journal of Phytopathology, 144(9-10), 449-454. http://dx.doi.org/10.1111/j.1439-0434.1996.tb00322.x 
Instituto Adolfo Lutz - IAL. (1985). Normas analíticas do Instituto Adolfo Lutz: Métodos químicos e físico para análise de alimentos (Vol. 1). São Paulo: IAL.

Jesus, E. R., Ellensohn, R. M., \& Smanioto, B. C. S. (2015). Óleo essencial de Melaleuca Alternifolia: Otimização do método analítico. UNOPAR Científica Ciências Exatas e Tecnológicas, 6(1), 67-72.

Keen, N. T., \& Yoshikawa, M. (1983). $\beta-1,3-E n d o g l u c a n a s e$ from soybean releases elicitoractive carbohydrates from fungal cell walls. Plant Physiology, 71(3), 460-465. PMid:16662849. http://dx.doi.org/10.1104/pp.71.3.460

Lajili, S., Azouaou, S. A., Turki, M., Muller, C. D., \& Bouraoui, A. (2016). Anti-inflammatory, analgesic activities and gastroprotective effects of the phenolic contents of the red alga, Laurencia obtusa. European Journal of Integrative Medicine, $8(3)$, 298-306. http://dx.doi.org/10.1016/j.eujim.2015.12.006

Lazazzara, V., Bueschl, C., Parich, A., Pertot, I., Schuhmacher, R., \& Perazzolli, M. (2018). Downy mildew symptoms on grapevines can be reduced by volatile organic compounds of resistant genotypes. Scientific Reports, 8(1), 1618. PMid:29374187. http://dx.doi.org/10.1038/s41598-018-19776-2

Lou, Z., Chen, J., Yu, F., Wang, H., Kou, X., Ma, C., \& Zhu, S. (2017). The antioxidant, antibacterial, antibiofilm activity of essential oil from Citrus medica L. var. sarcodactylis and its nanoemulsion. Lebensmittel-Wissenschaft + Technologie, 80, 371377. http://dx.doi.org/10.1016/j.Iwt.2017.02.037

Mazaro, S. M., Citadin, I., De Gouvêa, A., Luckmann, D., \& Guimarães, S. S. (2008). Indução de fitoalexinas em cotilédones de soja em resposta a derivados de folhas de pitangueira. Ciência Rural, 38(7), 1824-1829. http://dx.doi.org/10.1590/S010384782008000700004

Menolli, L. N., Finger, F. L., Puiatti, M., Barbosa, J. M., \& Barros, R. S. (2008). Atuação das enzimas oxidativas no escurecimento causado pela injúria por frio em raízes de batata-baroa. Acta Scientiarum. Agronomy, 30(1), 57-63.

Moraes, I. V. M., Cenci, S. A., Benedetti, B. C., Mamede, A. M. G. N., Soares, A. G., \& Barboza, H. T. G. (2008).Physical and chemical characteristics of minimally processed strawberries stored under refrigeration and controlled atmospheres. Science Technology Food, 28(2), 274-281. http://dx.doi.org/10.1590/S0101-20612008000200003

Neuhaus, J. M., Fritig, B., Linthorst, H. J. M., Meins, F., Mikkelsen, J. D., \& Ryals, J. (1996). A revised nomenclature for chitinase genes. Plant Molecular Biology Reporter, 14(2), 102-104. http://dx.doi.org/10.1007/BF02684897

Pârvu, M., Pârvu, A. E., Barbu-Tudoran, L., Roşca-Casian, O., Vlase, L., \& Danciu, M. R. (2013). In vitro effects of Allium obliquum extract on the growth and ultrastructure of Botrytis paeoniae. Journal of Medicinal Plants Research, 7(17), 1138-1145.

Pascholati, S. F., Leite, B., Stangarlin, J. R., \& Cia, P. (2008). Interação planta-patógeno: Fisiologia, bioquímica e biologia molecular. Piracicaba: FEALQ.

Piati, A., Holanda, N. M., \& Schneider, C. F. (2017). Efeito do óleo essencial de eucalipto sobre Penicillium digitatum. Revista Acadêmica: Ciência Animal, 11, 19-26.

Pommer, C. V., Passos, I. R. S., Terra, M. M., \& Pires, E. J. P. (1997). Variedades de videira para o Estado de São Paulo (Boletim Técnico, 166). Campinas: IAC.

Rasulov, A. T. (2017). Growing of high-qualitative table grapes for storage and transportation. Annals of Agrarian Science, 15(4), 439-442. http://dx.doi.org/10.1016/j.aasci.2017.02.016

Rodrigues, A. A. C., Bezerra Neto, E., \& Coelho, R. S. B. (2006). Indução de resistência a Fusarium oxysporum f. sp. tracheiphilum em caupi: Eficiência de indutores abióticos e atividade enzimática elicitada. Fitopatologia Brasileira, 31(5), 492499. http://dx.doi.org/10.1590/S0100-41582006000500009

Romani, L. (2011). Immunity to fungal infections. Nature Reviews. Immunology, 11(4), 275-288. PMid:21394104. http://dx.doi.org/10.1038/nri2939

Rossi Junior, J. A., \& Singleton, V. L. (1965). Colorimetry of total phenolics with phosphomolybdic phosphotungstic acid reagents. American Journal of Enology and Viticulture, 16, 144-158.

Sellamuthu, P. S., Sivakumar, D., Soundy, P., \& Korsten, L. (2013). Essential oil vapours suppress the development of anthracnose and enhance defence related and antioxidant enzyme activities in avocado fruit. Postharvest Biology and Technology, 81, 66-72. http://dx.doi.org/10.1016/j.postharvbio.2013.02.007

Shalaby, S., \& Horwitz, B. (2015). Plant phenolic compounds and oxidative stress: Integrated signals in fungal-plant interactions. Current Genetics, 61(3), 347-357. PMid:25407462. http://dx.doi.org/10.1007/s00294-014-0458-6

Shao, X., Cheng, S., Wang, H., Yu, D., \& Mungai, C. (2013). The possible mechanism of antifungal action of tea tree oil on Botrytis cinerea. Journal of Applied Microbiology, 114(6), 1642-1649. PMid:23495848. http://dx.doi.org/10.1111/jam.12193

Sivakumar, D., \& Bautista-Baños, S. (2014). A review on the use of essential oils for postharvest decay control and maintenance of fruit quality during storage. Crop Protection, 64, 27-37. http://dx.doi.org/10.1016/j.cropro.2014.05.012

Souza, C. F., Baldissera, M. D., Santos, R. C. V., Raffin, R. P., \& Baldisserotto, B. (2017). Nanotechnology improves the therapeutic efficacy of Melaleuca alternifolia essential oil in experimentally infected Rhamdia quelen with Pseudomonas aeruginosa. Aquaculture, 473, 169-171. http://dx.doi.org/10.1016/j.aquaculture.2017.02.014

Thambi, M., Tava, A., Mohanakrishnan, M., Subburaj, M., Pradeepkumar, K. M., \& Shafi, P. M. (2013). Composition and antimicrobial activities of the essential oil from Eugenia uniflora L. leaves growing in India. Journal of Pharmaceutical and Biomedical Sciences, 4, 46-49.

Van Loon, L. C. (2006). Regulation of changes in proteins and enzymes associated with active defense againt virus infections. In R. K. S. Wood (Ed.), Active mechanisms in plants (pp. 247-273). New York: Plenum Press. 
Óleos essenciais no controle de Botrytis cinerea: influência na qualidade pós-colheita de uvas 'Rubi'

Garcia, C. et al.

Zhang, Q., Zhou, W., Tan, H., Yang, M., \& Liu, X. (2016). Effects of coating and heat treatments on energy levels and physiological indexes and qualities of grape fruits during storage. Nongye Gongcheng Xuebao, 2(9), 255-263.

Zhang, X., \& Liu, C. (2015). Multifaceted regulations of gateway enzyme phenylalanine ammonia-lyase in the biosynthesis of phenylpropanoids. Molecular Plant, 8(1), 17-27. PMid:25578269. http://dx.doi.org/10.1016/j.molp.2014.11.001

Zimdars, S., Hitschler, J., Schieber, A., \& Weber, F. (2017). Oxidation of wine polyphenols by secretomes of wild Botrytis cinerea strains from white and red grape varieties and determination of their specific laccase activity. Journal of Agricultural and Food Chemistry, 65(48), 10582-10590. PMid:29125293. http://dx.doi.org/10.1021/acs.jafc.7b04375

Zoffoli, J. P., Latorre, B. A., Rodríguez, J., \& Aguilera, J. M. (2009). Biological indicators to estimate the prevalence of gray mold and hairline cracks on table grapes cv. Thompson Seedless after cold storage. Postharvest Biology and Technology, 52(1), 126133. http://dx.doi.org/10.1016/j.postharvbio.2008.11.010

Financiamento: Nenhum. 